\title{
Plasmalemma Vesicle-Associated Protein Has a Key Role in Blood-Retinal Barrier Loss
}

Joanna Wisniewska-Kruk, ${ }^{*}$ Anne-Eva van der Wijk, ${ }^{*}$ Henk A. van Veen, ${ }^{\dagger}$ Theo G.M.F. Gorgels, ${ }^{\ddagger}$ Ilse M.C. Vogels, ${ }^{*}$ Danielle Versteeg, ${ }^{\S}$ Cornelis J.F. Van Noorden, ${ }^{* \dagger}$ Reinier 0. Schlingemann, ${ }^{*}$ and Ingeborg Klaassen*

From the Ocular Angiogenesis Group, * Departments of Ophthalmology and Cell Biology and Histology, and the Core Facility Cellular Imaging, ${ }^{\dagger}$ Department of Cell Biology and Histology, Academic Medical Center, University of Amsterdam, Amsterdam; the University Eye Clinic Maastricht,${ }^{\ddagger}$ Maastricht University Medical Center, Maastricht; and the Hubrecht Laboratory and Interuniversity Cardiology Institute Netherlands, ${ }^{\S}$ Royal Netherlands Academy of Arts and Sciences, Utrecht, the Netherlands

Accepted for publication November 19, 2015.

Address correspondence to Ingeborg Klaassen, Ph.D., Ocular Angiogenesis Group, Departments of Ophthalmology and Cell Biology and Histology, Academic Medical Center, Meibergdreef 15 , Room L3-154, 1105 AZ Amsterdam, the Netherlands. E-mail: i.klaassen@ amc.uva.nl.

\begin{abstract}
Loss of blood-retinal barrier (BRB) properties induced by vascular endothelial growth factor (VEGF) and other factors is an important cause of diabetic macular edema. Previously, we found that the presence of plasmalemma vesicle-associated protein (PLVAP) in retinal capillaries associates with loss of BRB properties and correlates with increased vascular permeability in diabetic macular edema. In this study, we investigated whether absence of PLVAP protects the BRB from VEGFinduced permeability. We used lentiviral-delivered shRNA or siRNA to inhibit PLVAP expression. The barrier properties of in vitro BRB models were assessed by measuring transendothelial electrical resistance, permeability of differently sized tracers, and the presence of endothelial junction complexes. The effect of VEGF on caveolae formation was studied in human retinal explants. BRB loss in vivo was studied in the mouse oxygen-induced retinopathy model. The inhibition of PLVAP expression resulted in decreased VEGF-induced BRB permeability of fluorescent tracers, both in vivo and in vitro. PLVAP inhibition attenuated transendothelial electrical resistance reduction induced by VEGF in BRB models in vitro and significantly increased transendothelial electrical resistance of the nonbarrier human umbilical vein endothelial cells. Furthermore, PLVAP knockdown prevented VEGFinduced caveolae formation in retinal explants but did not rescue VEGF-induced alterations in endothelial junction complexes. In conclusion, PLVAP is an essential cofactor in VEGF-induced BRB permeability and may become an interesting novel target for diabetic macular edema therapy. (Am J Pathol 2016, 186: 1044-1054; http://dx.doi.org/10.1016/j.ajpath.2015.11.019)
\end{abstract}

Diabetic macular edema (DME) is the most frequent cause of vision loss among patients with diabetic retinopathy (DR). DME is a complex disease that has been associated with increased vascular permeability due to loss of the inner blood-retinal barrier (BRB). ${ }^{1}$ Disruption of the BRB leads to abnormal inflow of proteins and fluids into retinal tissue, which results in thickening of the central fovea and loss of visual acuity. Vascular endothelial growth factor (VEGF)-A, a potent inducer of vasopermeability and angiogenesis, is a major mediator in this process. ${ }^{2-4}$

The inner BRB is composed of endothelial cells, pericytes, and astrocytes, which form the neurovascular unit. ${ }^{1,5}$ The robust interactions among these BRB components are crucial for formation and maintenance of a physical and biochemical barrier between the retina and the vasculature. ${ }^{6-8}$ Despite the fact that the mechanisms of BRB breakdown in DR are multifactorial $^{9}$ and affect all components of the neurovascular unit, ${ }^{10-12}$ the most prominent pathologic changes are observed in endothelial cells. ${ }^{13-15}$ To date, two

Supported by Landelijke Stichting voor Blinden en Slechtzienden, Stichting Oogfonds Nederland, Stichting Winckel Sweep, UitZicht grant 2012-16, Nederlandse Vereniging ter Verbetering van het Lot der Blinden, Rotterdamse Stichting Blindenbelangen grant B20120030, Stichting Blindenhulp, and Stichting Nederlands Oogheelkundig Onderzoek grant 2012-05. This study was published with the help of Edward en Marianne Blaauw Fonds voor oogheelkunde (Edward and Marianne Blaauw fund for ophthalmology).

R.O.S. and I.K. contributed equally to this work as senior authors.

Disclosures: None declared.

Current address of J.W.-K., Karolinska Institutet, Department of Medical Biochemistry and Biophysics, Division of Vascular Biology, Stockholm, Sweden. 
mechanisms are known to contribute to VEGF-induced endothelial cell permeability: increased paracellular transport that involves changes in endothelial junction integrity and increased transcellular transport in endothelial cells mediated by caveolae. ${ }^{10,13-15}$ The effects of VEGF on altered tight junction integrity and decreased junctional protein expression have been extensively studied, $, 5,10,13,16,17$ but the effect of augmented caveolae formation in endothelium on BRB permeability remains not fully understood.

Plasmalemma vesicle-associated protein (PLVAP, PV-1) is an endothelial cell-specific protein ${ }^{18,19}$ that is known to be a structural component of caveolae, transendothelial channels, and fenestrae. ${ }^{20,21}$ PLVAP is widely expressed in capillaries and the venous vasculature but is absent in intact BRB and blood-brain barrier (BBB) endothelia. ${ }^{22-27}$ However, in pathologic conditions, such as $\mathrm{DR},{ }^{22,23,28}$ ischemia, or cancer, ${ }^{24,26,27}$ PLVAP expression in the BRB and $\mathrm{BBB}$ is highly up-regulated and associated with increased barrier permeability. ${ }^{23,25}$ In addition, PLVAP expression has been described to be present in immature and incompletely developed vasculature of the BRB and BBB. ${ }^{29-32}$ Recently, we studied the Akimba mouse, a model of advanced DR with hyperglycemia and high levels of intraocular VEGF, in which we found a correlation between Plvap mRNA levels and the degree of fluorescein leakage. ${ }^{10}$ PLVAP expression in endothelium is triggered by VEGF $^{6,13,26,28,33}$ in a VEGF receptor (VEGFR)2-dependent manner. ${ }^{26}$ Therefore, PLVAP may be involved in VEGF-induced BRB loss in DR.

Although PLVAP expression has been associated with BRB loss, a functional contribution of this protein to increased vascular permeability has not yet been found. This study examines the role of PLVAP in BRB loss in vivo and in vitro. To this end, we have assessed the changes that occur in permeability and in endothelial junctions and caveolae formation after knocking down PLVAP to elucidate the mechanisms that underlie the regulatory role of PLVAP in vascular permeability.

\section{Materials and Methods}

\section{Lentiviral Constructs}

For knockdown of PLVAP expression, shRNA lentiviral pLKO.1 constructs (SIGMA/TRC; Sigma-Aldrich, Zwijndrecht, the Netherlands) were used. Control cells were transduced with nontargeting shRNA (MISSION Non-Target shRNA Control Vector; Sigma-Aldrich). Virus particles were generated by co-transfecting the constructs with three packaging plasmids, pMDLg/pRRE, pMD2G, and RSV-Rev (Addgene, Cambridge, MA), into 293T cells. The sequences of PLVAP shRNA and Non-Target shRNA were 5'-CCGGCCCTTTCACACACACTTTCTACTCGAGTAGAAAGTGTGTGTGAAAGGGTTTTTTG-3' and 5'-CCGGCAACAAGATGAAGAGCACCAACTCGAGTTGGTGCTCTTCATCTTGTTGTTTTT-3', respectively.

\section{In Vitro BRB Model}

Cells were isolated as described previously. ${ }^{6}$ Three different models were assembled to study endothelial barrier permeability, including i) a bovine retinal endothelial cell (BREC) monolayer, ii) a triple co-culture model with BRECs, pericytes, and astrocytes, and iii) human umbilical vein endothelial cells (HUVECs). In the triple co-culture model, BRECs were seeded on top of the Transwell filter, primary rat astrocytes were seeded on the reverse side of the Transwell filters, and bovine primary pericytes were cultured on the bottom of a 24-well plate in which a Transwell filter was placed, as described previously. ${ }^{6}$ Three independent experiments were performed for each model $(n \geq 11)$.

\section{Permeability Assay}

Three days after assembling each of the models, shRNA lentiviral particles $(50 \mathrm{pg} / \mathrm{mL})$ were added to the cells. After 24 hours, cells were stimulated apically with $200 \mathrm{ng} / \mathrm{mL}$ human recombinant VEGF-A (Sanquin, Amsterdam, the Netherlands). Permeability was measured 72 hours after stimulation by adding 766 Da Cy3 (GE Healthcare, Eindhoven, the Netherlands) and $70 \mathrm{kDa}$ of fluorescein isothiocyanate (FITC)-dextran (FD; Sigma-Aldrich). Concentrations of the tracer molecules were determined using a fluorescence plate reader (BMG POLARstar; MTX Lab Systems, Vienna, VA), as described previously. ${ }^{6}$ Transendothelial electrical resistance (TEER) was measured in real time with the CellZscope system (NanoAnalytics, Münster, Germany) and expressed as $\Omega \cdot \mathrm{cm}^{2}$. In all experimental conditions, data were collected in quadruplicate. Three independent experiments were performed for each model.

\section{RNA Isolation and Real-Time PCR}

Total RNA was isolated using TRIzol reagent (Life Technologies, Bleiswijk, the Netherlands) following the manufacturer's protocol. Total RNA $(1 \mu \mathrm{g})$ was treated with DNase I (Amplification Grade; Life Technologies) and reverse transcribed into first-strand cDNA using a Maxima First Strand cDNA Synthesis Kit (Thermo Scientific, Roskilde, Denmark). ${ }^{6}$ Real-time PCR was performed using a CFX96 system (BioRad; Hercules, CA) as described previously. ${ }^{13}$ Expression data were normalized by the geometric mean of the two most stable housekeeping genes (ACTG1 and GAPDH), as determined by NormFinder. ${ }^{34}$ Three independent experiments were performed for each model $(n \geq 6)$. Primer sequences for bovine PLVAP were described previously. ${ }^{13}$ The primer sequences for ACTG1 and GAPDH were as follows: ACTG1, forward $5^{\prime}$-GATCTGGCACCACACCTTTT- ${ }^{\prime}$, reverse 5'-CCACATACATGGCAGGAGTG-3'; GAPDH, forward 5'-GGCGTGAACCACGAGAAGTATAA-3' ${ }^{\prime}$, reverse 5'-CCCTCCACGATGCCAAAGT-3'. 


\section{Oxygen-Induced Retinopathy Mouse Model}

Animal experiments were performed with the approval of the Animal Ethics Committee of the University of Amsterdam and in compliance with the Association for Research in Vision and Ophthalmology statement for the Use of Animals in Ophthalmic and Vision Research. From postnatal day 7 (P7), C57BL/6 mice were exposed to $75 \%$ oxygen for 5 days. ${ }^{35}$ At P12, pups were divided randomly, anesthetized, and injected intraocularly with $1 \mu \mathrm{L}$ of $50 \mu \mathrm{M}$ anti-Plvap siRNA (Accell SMARTpool; Thermo Scientific), treated with control siRNA (Accell Green Non-targeting siRNA; Thermo Scientific), or remained untreated. Pups were then returned to room air. At P17, fluorescein angiography was performed, and alternatively, eyes were analyzed for PLVAP expression and vascular leakage indicated by $\mathrm{IgG}$ extravasation into retinal tissue. Two independent experiments were performed $(n \geq 6)$.

For evaluation of blood vessel tortuosity, retinas were flat mounted and stained with isolectin IB4 probe conjugated with Alexa647 (Life Technologies). A minimum total of four blood vessels (arterioles and venules) were measured per retina ( $n \geq 8$ per group) using ImageJ version $1.48 \mathrm{v}$ (NIH, Bethesda, MD; http://imagej.nih.gov/ij). The tortuosity index was expressed as a quotient: vessel curve length (mean length, 788 $\mu \mathrm{m}$ ) over the line distance between the two ends.

\section{Fluorescein Angiography}

At P17, oxygen-induced retinopathy (OIR) mice were anesthetized. The pupils of the mouse eyes were dilated with tropicamide eye drops (Mydriacyl; Alcon Laboratories, Fort Worth, TX). A plano contact lens (Cantor + Nissel, Brackley, UK) was placed on the mouse eye to prevent dehydration of the cornea. Next, mice were injected intracardially with a mixture of $70 \mathrm{kDa} F D(10 \mathrm{mg} / \mathrm{mL}$; Sigma-Aldrich) and sodium fluorescein [approximately $4 \mathrm{kDa}, 10 \%$ in phosphatebuffered saline (PBS); Sigma-Aldrich]. Immediately after injections, image acquisition was started using the scanning laser ophthalmoscope HRA2 (Heidelberg Engineering, Heidelberg, Germany). The HRA2 was operated in the fluorescence mode with the excitation light provided by a 488-nm Argon laser. All images were acquired by using the automatic real-time mode. Eyes were enucleated 20 minutes after perfusion of tracers, dissected, and fixed in $4 \%$ paraformaldehyde for 4 hours at $4^{\circ} \mathrm{C}$. Next, retinas were washed in PBS to remove sodium fluorescein from the tissue and flat mounted. Images of retinas were recorded using a wide-field fluorescence microscope (Leica, Mannheim, Germany). The images were processed in GIMP software version 2.8v (GNU Image Manipulation Program; GNOME Foundation, Cambridge, MA; http://www.gimp.org).

\section{Fluorescence Immunohistochemistry}

Mouse retinas were permeabilized with $0.5 \%$ Triton X-100 for 30 minutes and incubated for 1 hour with $2 \%$ normal goat serum (Dako, Glostrup, Denmark). Subsequently, retinas were incubated overnight at $4{ }^{\circ} \mathrm{C}$ with isolectin IB4 (Alexa 546 conjugated; Invitrogen), anti-PLVAP antibody (rat monoclonal, MECA-32; Abcam, Cambridge, UK), or antimouse IgG antibody (Dako). Next, retinas were washed three times for 30 minutes in PBS, and Cy3-, Cy5-, FITC-, or Alexa488-conjugated secondary antibodies (Jackson ImmunoResearch, Suffolk, UK) were added. After 2 hours of incubation, retinas were washed three times for 30 minutes in PBS. Finally, retinas were flat mounted and covered with fluorescence mounting medium (Dako).

BRECs or HUVECs were cultured on collagen- or gelatincoated plastic coverslips (Nunc, Thermo Scientific), respectively. Cells were fixed and stained as described previously. ${ }^{6}$ The following antibodies were used: anti-claudin-5 (rabbit polyclonal; Life Technologies), anti-vascular endothelial cadherin (rabbit polyclonal; Abcam), anti-zonula occludens protein 1 (rabbit polyclonal; Life Technologies), anti-VEGFR2 (rabbit polyclonal; Abcam), phalloidin (Texas Red-X; Invitrogen, Life Technologies), and anti-PLVAP (174/2; Abcam). Nuclei were stained using $1 \mu \mathrm{g} / \mathrm{mL}$ Hoechst dye (Life Technologies). Images were recorded using a confocal laser scanning microscope SP8 (Leica). In all experiments, specificity of the staining was tested by omitting primary antibody. Three independent experiments were performed $(n \geq 8)$.

To quantify actin stress fibers, GIMP software was used. Images of BRECs stained with phalloidin probe were converted to gray scale images with three shades of gray. Stress fibers were assessed by quantifying only the brightest pixels divided by the total amount of gray pixels, representing the amount of total fiber.

\section{Cell-Based Enzyme-Linked Immunosorbent Assay}

BRECs were seeded in a 96-well plate (Corning, Canton, MA). After reaching confluence, cells were transduced with lentiviral constructs and 24 hours later stimulated with $200 \mathrm{ng} /$ $\mathrm{mL}$ human recombinant VEGF. Cells were fixed with $4 \%$ paraformaldehyde and stained as described previously. ${ }^{6}$ The following primary antibodies were used: anti-claudin-5 (rabbit polyclonal; Life Technologies), anti-vascular endothelial cadherin (rabbit polyclonal; Abcam), and anti-occludin (mouse monoclonal; Life Technologies). Primary antibodies were detected using horseradish peroxidase-conjugated antibody (rabbit anti-mouse and swine anti-rabbit antibody; Dako). Next 3,3',5,5'-tetramethylbenzidine (Merck, Whitehouse Station, $\mathrm{NJ}$ ) was added. The reaction was stopped with $1 \mathrm{M} \mathrm{H}_{2} \mathrm{SO}_{4}$ (Merck). The absorbance was measured on a plate reader (Bio-Rad) with excitation at $450 \mathrm{~nm}$ and emission at 655 $\mathrm{nm}$. Two independent experiments were performed $(n \geq 7)$.

\section{Electron Microscopy}

Human donor eyes were provided by the Euro Cornea Bank (Beverwijk, the Netherlands) after removal of corneal buttons for transplantation. The use of human material was in 

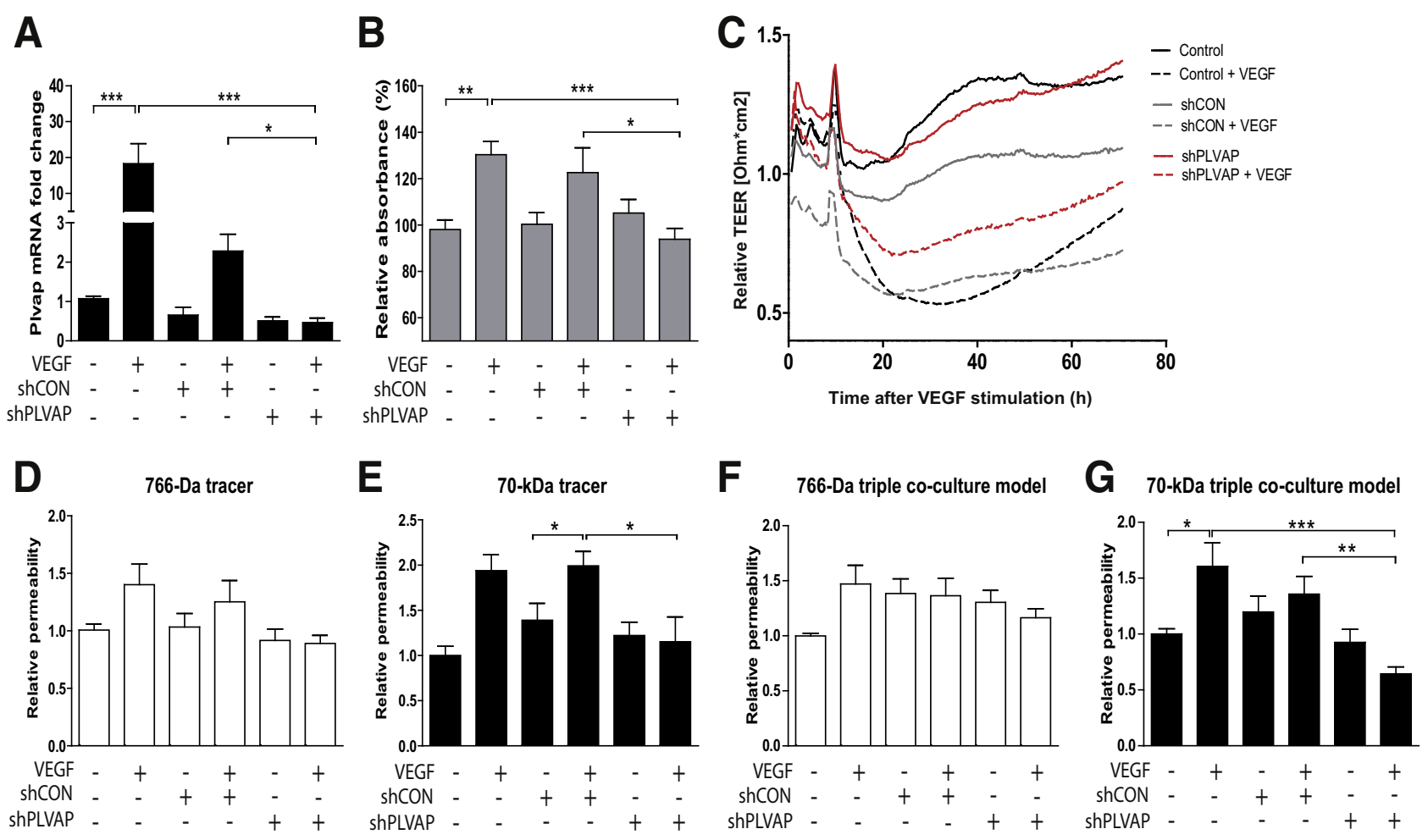

Figure 1 Inhibition of plasmalemma vesicle-associated protein (PLVAP) expression reduces vascular endothelial growth factor (VEGF)-induced permeability in the in vitro blood-retinal barrier (BRB) model. Relative PLVAP mRNA (A) and protein (B) levels in control and shRNA transduced bovine retinal endothelial cells (BRECS) that were cultured in the presence or absence of VEGF. mRNA expression was measured using real-time PCR, and protein levels were quantified using cell-based enzyme-linked immunosorbent assay. Lentiviral delivered shPLVAP constructs significantly block VEGF-induced PLVAP expression, both at the mRNA and protein levels. C: Changes in real-time transendothelial electrical resistance (TEER) in control (shCON) and shRNA (shPLVAP) expressing BRECs, cultured in the presence or absence of VEGF. Knockdown of PLVAP expression reduces the VEGF-induced decrease in TEER values. D and E: Relative permeability after knocking down PLVAP expression of 767-Da Cy3 (D) and 70-kDa dextran tracer (E) was measured in BREC monolayers. F and G: Relative permeability in the triple co-culture BRB model of 767-Da Cy3 (F) and 70-kDa dextran (G) after knocking down PLVAP in endothelial cells. Endothelial permeability in all BRB models was induced by $200 \mathrm{ng} / \mathrm{mL}$ VEGF for 72 hours. ${ }^{*} P<0.05,{ }^{*} P<0.01$, and ${ }^{* * *} P<0.001$.

accordance with the international declaration of Helsinki. The Euro Cornea Bank obtained permission from the donors for eye autopsy and the use of clinical information for research purposes. Retinas were dissected from donor eyes (57-year-old man and 62-year-old woman, with no clinical manifestations of diabetes or DR) and sampled using an 8-mm biopsy puncher (Stiefel, Watford, UK). Retinas were cultured in neurobasal medium (Gibco, Life Technologies) supplemented with B27 Serum Free Supplement (Gibco) and antibiotics (1\% penicillin streptomycin; Life Technologies). After overnight incubation with lentiviral shRNA constructs (short hairpin control and PLVAP), retinas were stimulated after 72 hours with $100 \mathrm{ng} / \mathrm{mL}$ human recombinant VEGF, and samples were fixed and stored in McDowell phosphate buffer. ${ }^{36}$ After fixation, the samples were washed in $0.1 \mathrm{M}$ phosphate buffer followed by washing in distilled water, osmication for 75 minutes in $1 \% \mathrm{OsO}_{4}$ in water, and additional washing in distilled water. For contrast enhancement in the electron microscope, biopsy specimens were block stained overnight in $1.5 \%$ aqueous uranyl acetate and then dehydrated through incubation in ethanol and embedded in epon 812 (Electron Microscopy Sciences, Hatfield, PA). The resin blocks were polymerized for 48 hours at $60^{\circ} \mathrm{C}$. Ultrathin sections of 90 nm were cut on a Reichert EM UC6 (Leica) with a diamond knife, collected on Formvar (Structure Probe Inc., West Chester, PA) coated grids, and stained with uranyl acetate and lead citrate. Micrographs were taken with a Veleta Transmission Electron Microscopy camera (Olympus, Soft Imaging Solutions, Münster, Germany). The images were masked and analyzed using iTEM software version 6076 (Olympus Soft Imaging Solutions). The number of caveolae in endothelial cells was assessed by counting the caveolae-like membrane invaginations on the luminal and abluminal side in retinal blood vessel. A mean of $28.5 \mu \mathrm{m}$ of endothelium, including luminal (mean $=13.3 \mu \mathrm{m}$ ) and abluminal $($ mean $=15.2 \mu \mathrm{m}$ ) linear length of blood vessel, was evaluated for the presence of caveolae $(n=15$ from two different donors).

\section{Statistical Analysis}

One-way analysis of variance followed by Bonferroni's multiple comparison test were used to evaluate the statistical significance of the data. Results are expressed as means \pm SEM. $P<0.05$ was considered significant. 
A
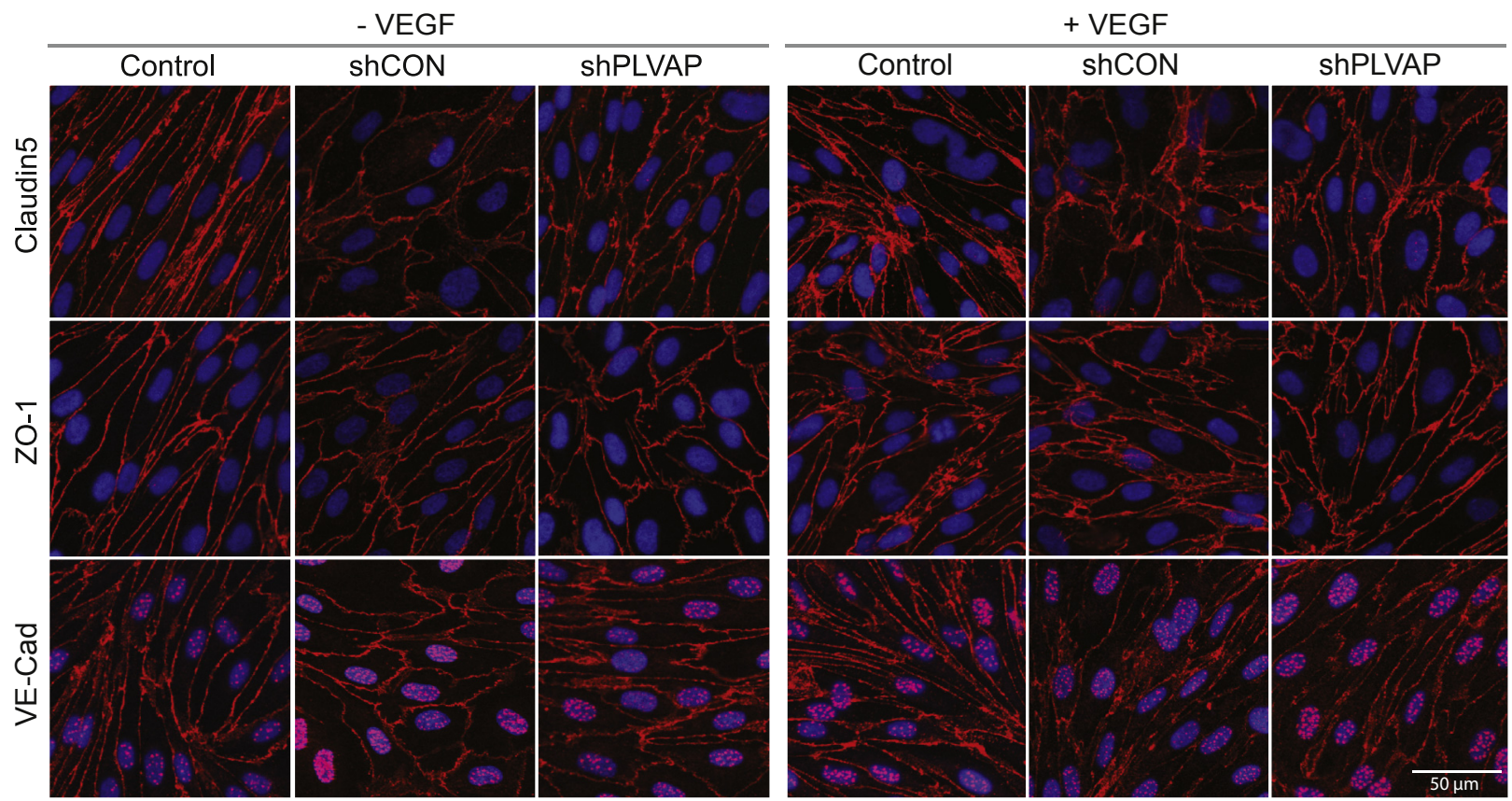

B

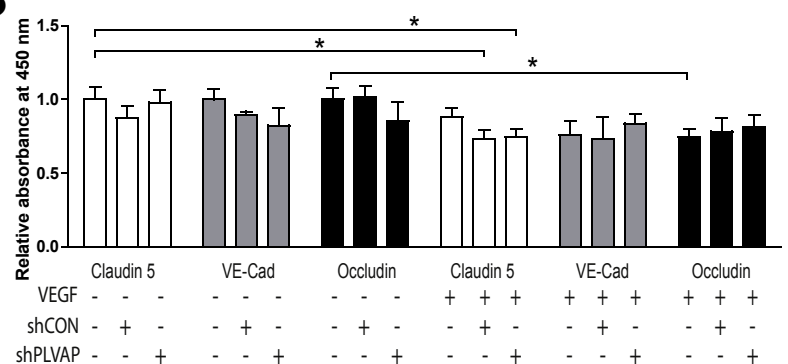

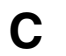

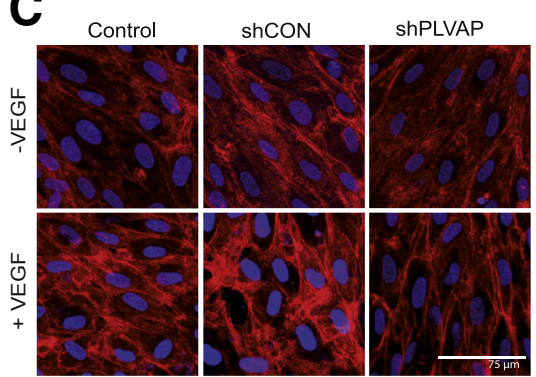

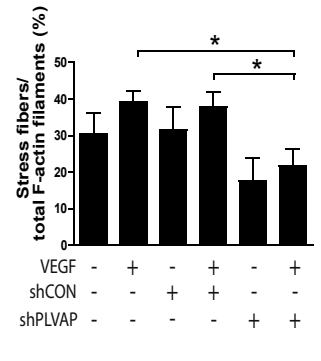

Figure 2 Knockdown of plasmalemma vesicle-associated protein (PLVAP) expression does not prevent vascular endothelial growth factor (VEGF)-induced alterations in tight junction complexes. A: The presence of $200 \mathrm{ng} / \mathrm{mL}$ VEGF leads to loss of tight junction integrity in bovine retinal endothelial cells (BRECs). Tight junction staining is presented in red and nuclear staining in blue. B: Inhibition of PLVAP expression has a modest effect on the preservation of the tight junction integrity and does not prevent down-regulation of tight junction protein expression in BRECs after VEGF stimulation (200 $\mathrm{ng} / \mathrm{mL}$ ), as determined by cell-based enzyme-linked immunosorbent assay. Protein levels and statistical comparisons are relative to untreated controls. C: Silencing of PLVAP expression in BRECs reduces VEGF-induced stress fiber formation. F-actin filaments were stained using the phalloidin probe. Stress fibers were selected on the basis of on staining intensity (as described in Materials and Methods). Images were recorded using confocal microscopy. Data is presented as means \pm SD. ${ }^{*} P<0.05$. VE-Cad, vascular endothelial cadherin; Z0-1, zonula occludens protein 1.

\section{Results}

\section{PLVAP Inhibition Reduces VEGF-Induced BRB Permeability in Vitro}

The endothelial barrier in our in vitro BRB model is formed by BRECs. The basal PLVAP expression in these cells is low, but stimulation with $200 \mathrm{ng} / \mathrm{mL}$ VEGF for 72 hours leads to significant induction of PLVAP expression, both at the mRNA (Figure 1A) and protein level (Figure 1B). Knocking down PLVAP significantly blocked VEGF-induced PLVAP expression (Figure 1, A and B), thus confirming an efficient inhibition by shRNA.

To study whether increased PLVAP expression in retinal capillaries is necessary for loss of BRB integrity, we knocked down PLVAP expression in BRECs and induced BRB breakdown using $200 \mathrm{ng} / \mathrm{mL}$ VEGF. As expected, stimulation of BRECs with VEGF resulted in a clear decrease in TEER values, which reflects the loss of endothelial barrier properties (Figure 1C). Knockdown of PLVAP expression in BRECs before VEGF stimulation considerably diminished the decrease in TEER values (Figure 1C), suggesting that the absence of PLVAP in endothelial cells has a protective effect on VEGFinduced BRB breakdown. Consistently, knockdown of PLVAP expression in BREC monolayers prevented VEGFinduced permeability for small 766-Da (Figure 1D) and large $70-\mathrm{kDa}$ (Figure 1E) fluorescent tracers 72 hours after stimulation. Given the fact that the BRB is formed by endothelial cells, pericytes, and astrocytes, ${ }^{1}$ we further investigated the effect of knocking down PLVAP on VEGF-induced permeability in the triple co-culture BRB model. Knockdown of PLVAP in the triple co-culture BRB model did not significantly reduce 


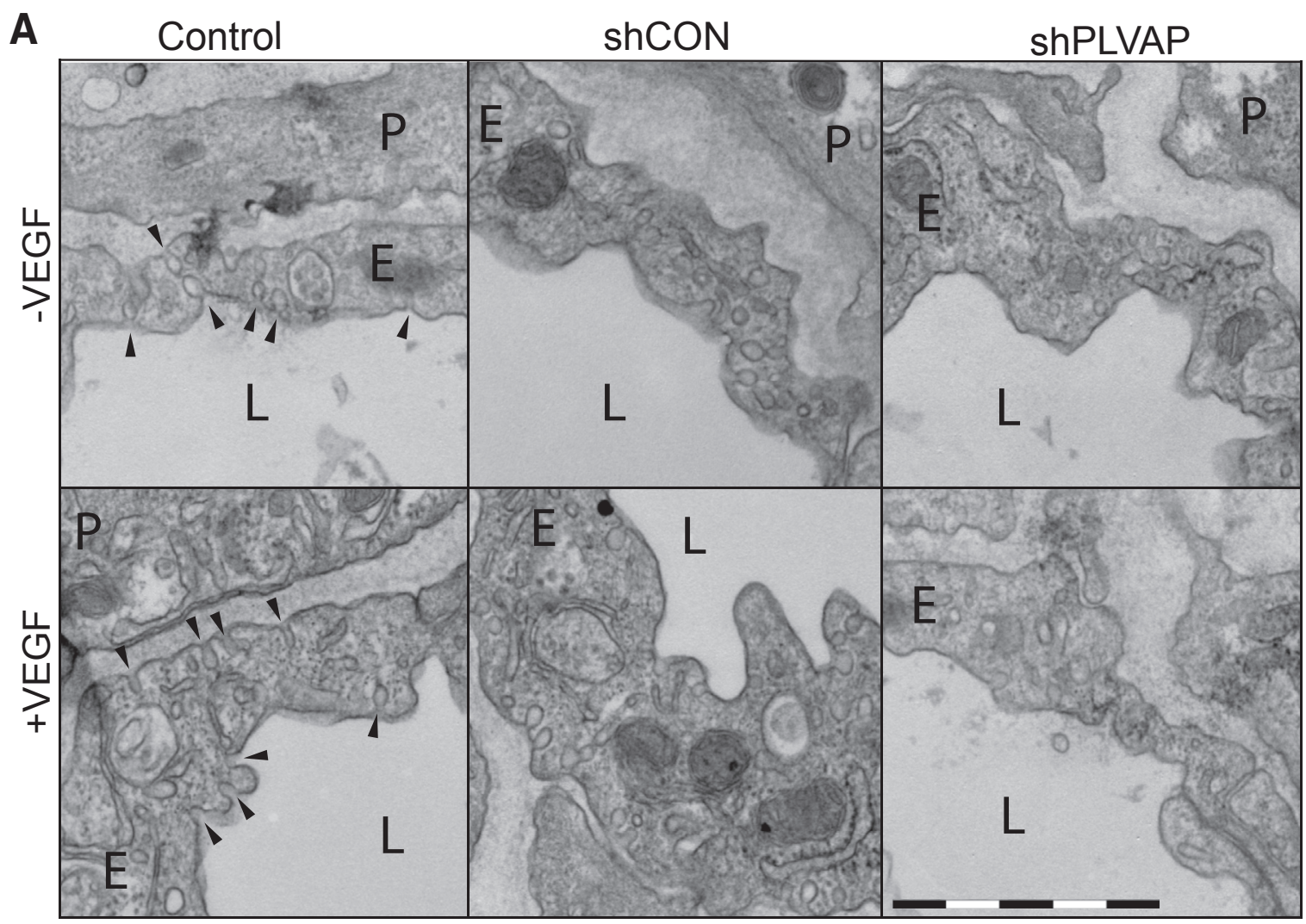

B

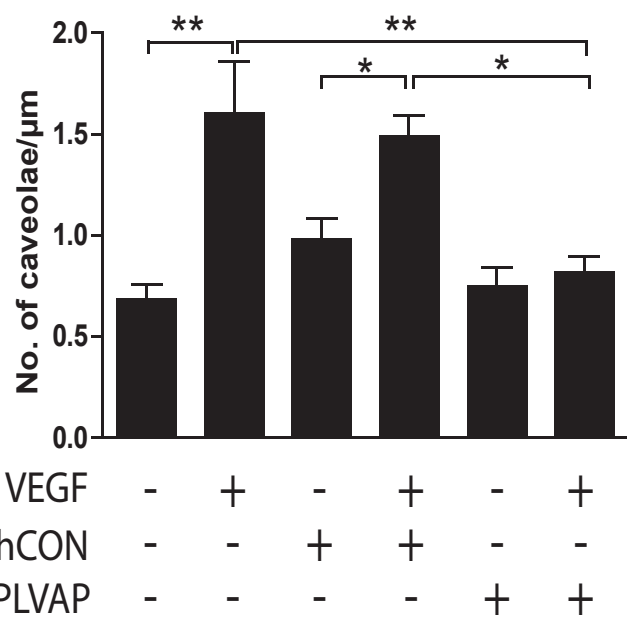

VEGF-induced permeability for 766-Da tracers (Figure 1F) but significantly inhibited the permeability for $70-\mathrm{kDa}$ molecules 72 hours after stimulation (Figure 1F). Overall, these data indicate that PLVAP is necessary for VEGF-induced BRB loss.

\section{Effect of PLVAP Inhibition on Endothelial Junction Protein Expression and Integrity}

On the basis of TEER and permeability measurements of BRECs cultured on Transwell filters in the presence of increasing doses of VEGF (data not shown), we decided to use a dose of $200 \mathrm{ng} / \mathrm{mL}$ for subsequent in vitro experiments. VEGF stimulation of BRECs for 12 hours resulted in endothelial junction disorganization along the cell periphery and
Figure 3 Inhibition of plasmalemma vesicleassociated protein (PLVAP) expression in human retinal explants blocks vascular endothelial growth factor (VEGF)induced caveolae formation in endothelial cells. A: Human retinal explants were transduced with shRNA constructs [control (shCON) and shRNA (shPLVAP)] and cultured in the presence or absence of $100 \mathrm{ng} / \mathrm{mL}$ VEGF for 72 hours. VEGF stimulation induced caveola formation in endothelial cells, whereas inhibition of PLVAP expression significantly blocked this process. Only caveolae-like invaginations on the luminal and abluminal side in retinal blood vessel were scored per linear micrometer of endothelium. Examples of caveolae are marked by black arrowheads. B: Quantification of caveolae in endothelial cells of blood vessels present in human retinal explants. ${ }^{*} P<0.05$, ${ }^{* *} P<0.01$. Scale bar $=1 \mu \mathrm{m}$. E, endothelial cell; $\mathrm{L}$, lumen of a blood vessel; $P$, pericyte.

increased cytoplasmic staining of claudin-5, zonula occludens protein 1, and vascular endothelial cadherin protein (Figure 2A), as determined by immunofluorescence. Furthermore, by using cell-based enzyme-linked immunosorbent assay, we found that VEGF induces a down-regulation of vascular endothelial cadherin and occludin expression in BRECs (Figure 2B). Knockdown of PLVAP expression had only a modest positive effect on the preservation of endothelial junction integrity (Figure 2A) and did not rescue them from VEGF-induced degradation (Figure 2B).

In our previous study, we found that VEGF-induced stress fiber formation in endothelial cells leads to the formation of gaps between endothelial cells, which may directly contribute to increased barrier permeability. ${ }^{6}$ 
A
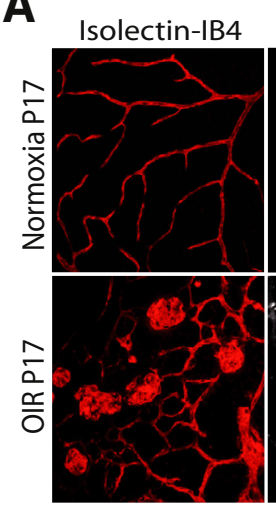

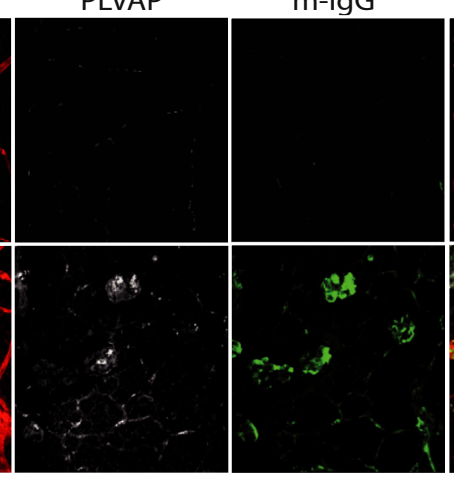

PLVAP
Merge

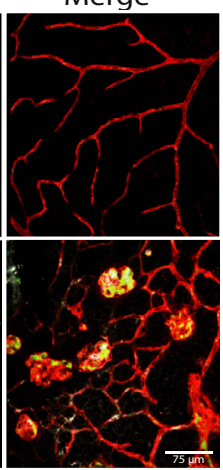

B

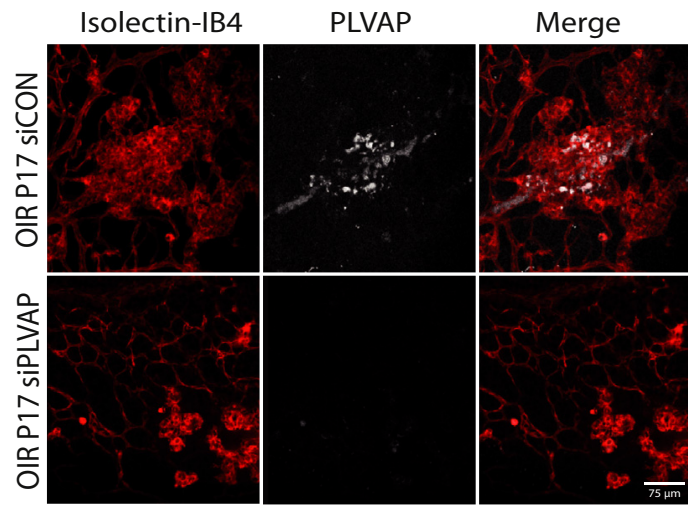

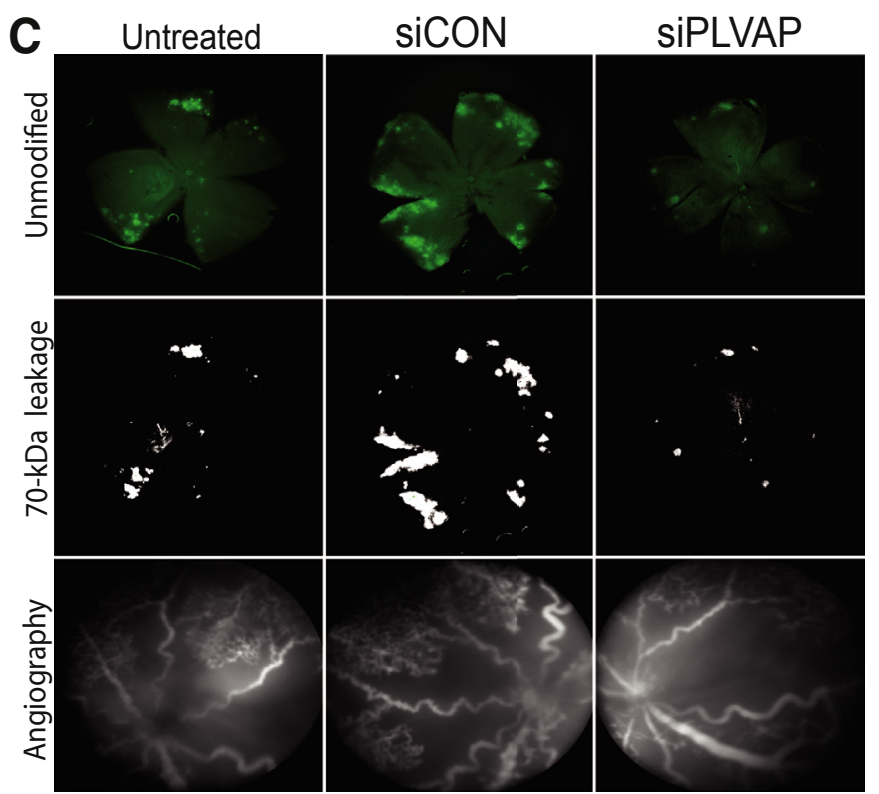

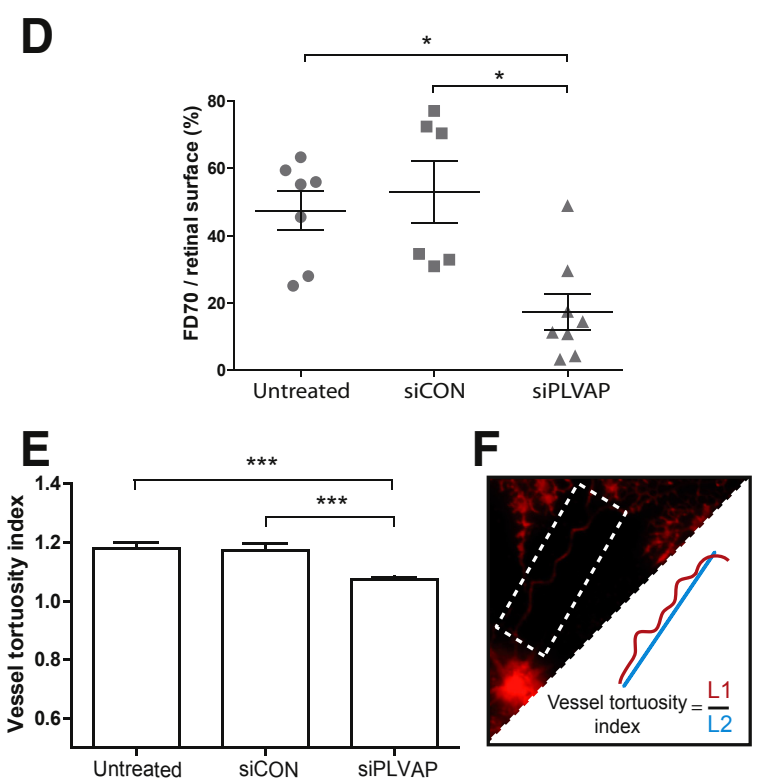

Figure 4 Knockdown of plasmalemma vesicle-associated protein (PLVAP) expression reduces blood-retinal barrier (BRB) leakage and tortuosity of blood vessels in oxygen-induced retinopathy (OIR). A: PLVAP expression and extravasation of endogenous mouse $\mathrm{IgG}$ (m-IgG), a marker of BRB permeability, increase in OIR retinas. Note the formation of vascular tufts as a result of pathological angiogenesis triggered by retinal hypoxia. B: Intraocular treatment with siRNA targeting Plvap results in significantly reduced PLVAP protein expression in the retinal vasculature in OIR compared with control nontargeting siRNA treatment (siCON). Images were recorded using confocal microscopy. C: Inhibition of Plvap expression in OIR retinas results in decreased extravasation of fluorescently labeled tracers into the retina (top row). Images were recorded using wide-field fluorescence microscopy. Examples of images representing tracer leakage are shown in the middle row. In addition, vascular leakage in OIR retinas was visualized by fluorescence angiography (bottom row). Images are representative for each experimental group. D: Quantification of fluorescently labeled tracers that leaked into the retina. The extravasation of the 70-kDa tracer into retinal tissue was quantified using graphic software. E: Inhibition of Plvap expression in OIR retinas results in decreased tortuosity of blood vessels. F: Blood vessel tortuosity index is expressed as a quotient: length of curved blood vessel over linear distance between ends. ${ }^{*} P<0.05,{ }^{* *} P<0.001$. Scale bar $=1000 \mu \mathrm{m}$ (B).

A significant reduction in stress fiber formation after inhibition of PLVAP expression was observed in VEGFstimulated BRECs (Figure 2C), which may contribute to the maintenance of the contact between endothelial cells and the barrier integrity.

\section{Knockdown of PLVAP Reduces the Number of} Endothelial Caveolae in Human Retinal Explants after VEGF Stimulation

To investigate the potential role of PLVAP in increased endothelial cell permeability via induction of caveolae-mediated transcellular transport in these cells, ex vivo cultured human retinas were transduced with shRNA and cultured in the presence or absence of $100 \mathrm{ng} / \mathrm{mL}$ VEGF. In control samples incubated with VEGF, a significantly increased number of caveolae was observed in endothelial cells (Figure 3A). Knockdown of PLVAP expression significantly blocked this VEGF-induced formation of caveolae (Figure 3B) but did not alter the basal levels of caveolae in endothelial cells. No differences in the ratio between luminal and abluminal number of caveolae was observed among all investigated conditions (data not shown). 
A
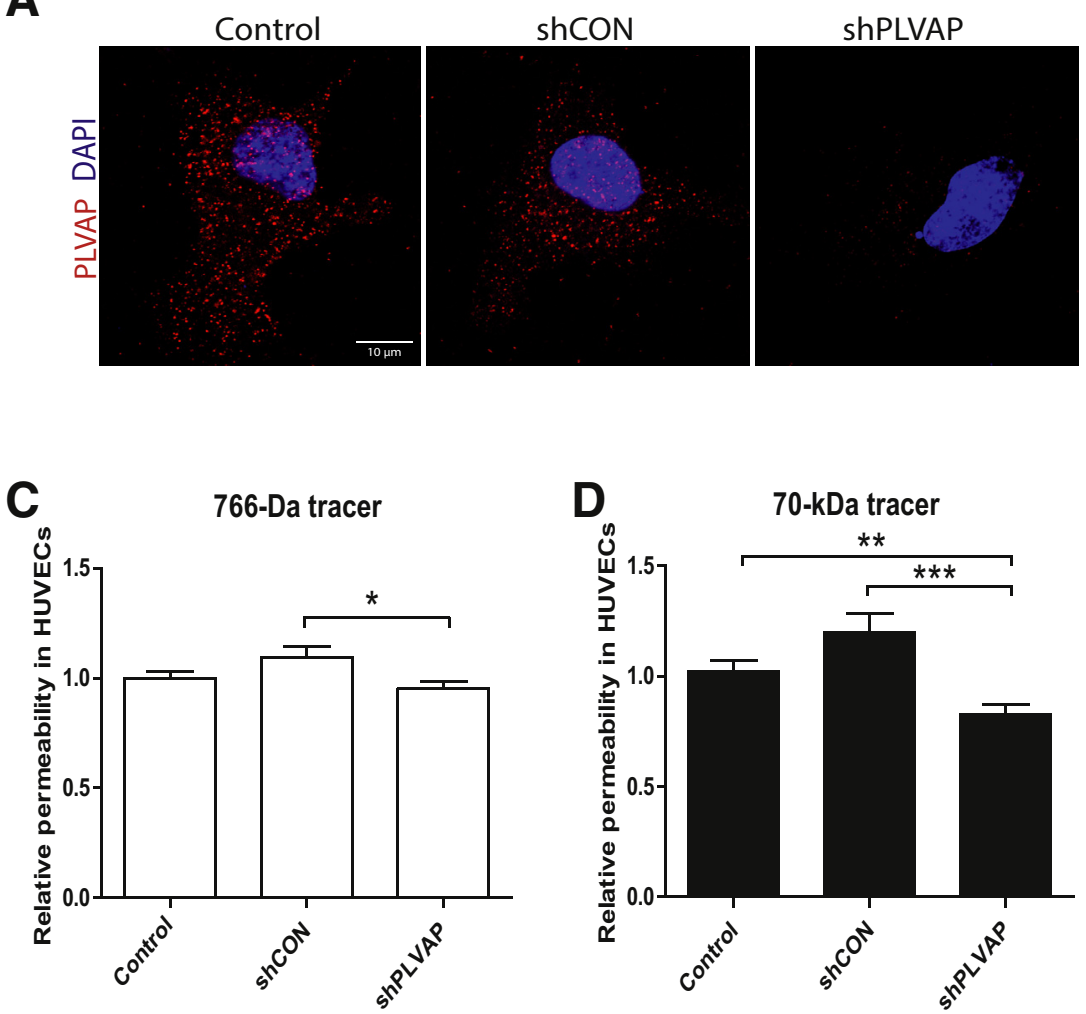

B

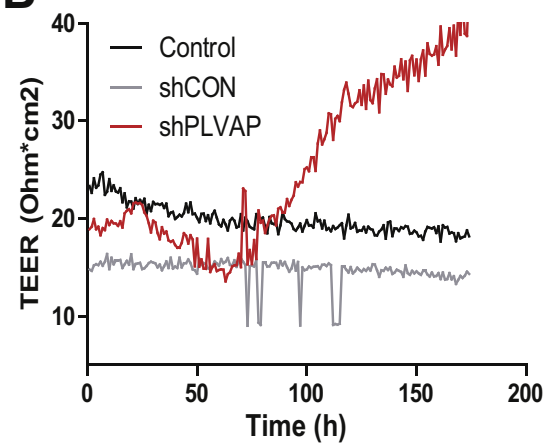

Figure 5 Blocking plasmalemma vesicleassociated protein (PLVAP) expression leads to barrier formation in nonbarrier type of endothelium. A: PLVAP protein expression in human umbilical vein endothelial cells (HUVECs). Images were recorded using confocal microscopy. B-D: Knockdown of PLVAP expression in HUVEC induces increased transendothelial electrical resistance (TEER) values (B) and decreased permeability for 766-Da (C) and 70-kDa (D) tracers. Permeability of HUVEC monolayers was measured 72 hours after lentiviral delivery of shRNA constructs. ${ }^{*} P<0.05$, $* * P<0.01$, and ${ }^{* * *} P<0.001$.

\section{Knockdown of PLVAP Reduces Hypoxia-Induced Retinal Vascular Leakage in Vivo}

To confirm the functional role of PLVAP in retinal vascular permeability in vivo, we evaluated the effects of knocking down Plvap expression in the mouse OIR model. In this model, P7 mouse pups are exposed to $75 \%$ oxygen until P12, which results in large capillary obliteration. Next, pups are removed from the hyperoxia and placed in normoxia, which leads to onset of hypoxia and an overexpression of hypoxia-induced growth factors, such as VEGF, which is a potent inducer of angiogenesis and vessel permeability.

In the OIR model, we observed both high PLVAP protein expression in endothelium and vascular leakage in the retina, as indicated by extravasation of endogenous IgG molecules (Figure 4A). In most samples, PLVAP expression was visibly up-regulated in the neovascular tufts, which are a hallmark of the OIR model. Immunofluorescence analysis of OIR retinas revealed that intraocular treatment with Plvap-targeting siRNA resulted in decreased PLVAP protein expression in retinal blood vessels at P17 compared with eyes treated with control siRNA (Figure 4B). Furthermore, knockdown of Plvap expression in OIR eyes significantly reduced retinal vascular leakage of 70-kDa FITC-labeled dextran (Figure 4, C and D) and tortuosity of blood vessels (Figure 4, E and F) compared with untreated eyes and eyes treated with control siRNA. Fluorescein angiography images of live animals revealed diffuse leakage of fluorescently labeled tracers (4 and $70 \mathrm{kDa}$ ) from preexisting retinal blood vessels with no significant differences among all experimental conditions (Figure 4C). Overall, these results indicate that PLVAP plays a critical role in hypoxia-driven increased vascular permeability and vascular tortuosity in the OIR model.

\section{PLVAP Knockdown Induces Barrier Properties in HUVECS}

In physiological conditions, PLVAP protein expression is absent from barrier endothelium, ${ }^{22,23,28}$ but it is widely expressed in other endothelia of the vascular system. ${ }^{18,37}$ Therefore, we hypothesized that inhibition of PLVAP expression in a nonbarrier type of endothelium, such as HUVECs, results in the formation of an endothelial barrier. HUVECs express considerable levels of PLVAP (Figure 5A) and form a relatively permeable barrier, as indicated by low TEER values (Figure 5B). Knockdown of PLVAP expression in HUVECs resulted in a twofold increase in TEER value (Figure 5B) and significantly reduced permeability for 766-Da (Figure 5C) and $70-\mathrm{kDa}$ fluorescent tracers (Figure 5D) compared with untreated HUVECs. These results indicate that PLVAP expression in nonbarrier endothelium is a prerequisite for a permeable endothelial functional barrier phenotype.

\section{Discussion}

Disruption of the BRB in patients with DR leads to leakage of plasma proteins and fluid from the microvasculature and 
subsequent retinal edema. Although the mechanisms responsible for BRB loss in DR are not completely understood, VEGF is one of the key mediators in this process. ${ }^{2,4}$ High VEGF levels induce PLVAP expression, ${ }^{26,28}$ which in physiological conditions is absent from the intact BRB. ${ }^{18,19,22,23}$ In organs with blood-tissue barriers, such as the brain and eye, the presence of PLVAP indicates the lack of a blood-tissue barrier ${ }^{22,25}$ and correlates with increased microvascular permeability in pathologic conditions, such as brain tumors, ${ }^{24,26,27}$ brain ischemia, ${ }^{25}$ and DR. ${ }^{23,28,38}$

We observed a significant reduction of VEGF-induced BRB permeability in vitro and of BRB loss in vivo after knockdown of PLVAP expression. VEGF disrupts the endothelial barrier by inducing tight junction phosphorylation and degradation $^{6,13-16,39}$ and increasing transendothelial transport mediated by caveolae. ${ }^{13,40-42}$ We hypothesized that PLVAP knockdown would mainly have an effect on transcellular transport because it is a structural component of caveolar diaphragms. ${ }^{21}$ Indeed, we observed in vitro the most prominent effect of PLVAP silencing on VEGF-induced permeability for large fluorescent tracers $(70 \mathrm{kDa})$, which we hypothesize to preferentially cross the endothelium via the transcellular pathway. Similarly, we observed in in vivo studies a significantly reduced leakage of the 70-kDa tracer, whereas no effect on leakage of the small molecule sodium fluorescein (approximately $4 \mathrm{kDa}$ ) was observed in mice injected with siRNA against Plvap in the OIR model. Furthermore, this finding is consistent with the finding that inhibition of PLVAP prevented the induction of caveolae by VEGF in human retinal explants. Our data on tight junction integrity further support the idea that inhibition of PLVAP mainly affects the transcellular pathway because VEGFinduced disruption of tight junctions could not be prevented by inhibition of PLVAP, despite a reduction in stress fiber formation.

Knockdown of PLVAP expression in human retinal explants blocked VEGF-induced caveolae formation but did not alter the basal levels of caveolae, which is in agreement with previous reports. ${ }^{43}$ This finding suggests that PLVAP is necessary for the formation of new caveolae in conditions with increased permeability. The finding that inhibition of PLVAP has a similar effect on caveolae formation and permeability in the presence of VEGF suggests that the effects of PLVAP on vascular permeability are explained by its structural role in caveolae. In our previous studies, we found that intraocular injections of VEGF in cynomolgus monkeys not only increased the expression of PLVAP protein $^{28}$ but also caused a shift in the distribution of caveolae from a predominantly abluminal localization to a predominantly luminal localization, similar to the situation in nonbarrier endothelium. ${ }^{42}$

VEGFR2 is the key mediator of VEGF signaling in endothelial cells ${ }^{2,44}$ and is localized in caveolae. ${ }^{45}$ Therefore, reduced numbers of caveolae may alter VEGF signaling. Coregulated expression of VEGFR2 and PLVAP was observed in previous studies. ${ }^{38}$ In healthy patients, in which the BRB is intact, low numbers of caveolae and low VEGFR2 and PLVAP protein levels were found. On the other hand, in DR both VEGFR2 and PLVAP are expressed in retinal capillaries with BRB loss as demonstrated by leakage of endogenous plasma proteins. ${ }^{23,33,38}$ Besides VEGFR2, caveolae harbor many other receptors involved in vascular permeability, including transforming growth factor- $\beta$ receptors, ${ }^{46}$ that may be reduced as well in the absence of PLVAP in endothelial cells. Consistent with this notion, we found in the present study that nonbarrier endothelial cells (HUVECs), which have constitutive expression of PLVAP, form a tighter endothelial barrier after knockdown of PLVAP expression. Hence, absence of PLVAP expression in physiologic conditions in the retina seems to be essential for maintaining BRB properties.

Outside the eye and brain, PLVAP is expressed in large and medium-sized veins and in continuous and fenestrated endothelia. ${ }^{18}$ In these fenestrations, PLVAP forms diaphragms that may work as a sieve to regulate nonspecific transport of plasma proteins. In PLVAP knockouts, increased vascular leakage ${ }^{40}$ may be explained by the absence of these fenestral sieves, whereas in VEGF-induced conditions, PLVAP may also regulate VEGFR2 availability and therefore also facilitate a VEGF-induced decrease in endothelial junctions. ${ }^{47}$ This finding may explain why plasma proteins in normal vascular beds and tumors have occasionally been found to extravasate paracellularily. ${ }^{48}$

Although our present and previous data clearly indicate a function of caveolae in VEGF-induced vascular permeability, ${ }^{1,10,13,42}$ the exact molecular function of caveolae in relation to PLVAP in BRB permeability remains incompletely understood and needs to be addressed in future studies. Our results indicate that PLVAP can be an interesting novel therapeutic target for the treatment of DME. This finding is further supported by our recent study in the Akimba mouse, ${ }^{10}$ a model that presents many clinical features of DR, including DME, ${ }^{11}$ in which we found a correlation between PLVAP levels and the degree of fluorescein leakage in the retina. The Akimba mouse was generated by crossing the Kimba mouse, which has high levels of intraocular VEGF, and the Akita mouse, which spontaneously develops diabetes. ${ }^{11}$ In the Kimba mouse, we found a strong but statistically insignificant correlation between PLVAP levels and the degree of fluorescein leakage, suggesting that other factors other than VEGF play a role in the induction of PLVAP.

The currently available clinical approaches to treat DR are laser surgery, corticosteroids, and anti-VEGF therapy. Because VEGF is involved in many physiological processes in the retina, such as neurogenesis, neuroprotection, glial growth, and endothelial cell survival, ${ }^{49}$ the long-term use of anti-VEGF compounds may potentially lead to serious adverse effects, such as photoreceptor degeneration ${ }^{11}$ and Müller cell death. ${ }^{50}$ In addition, VEGF-neutralizing antibodies in proliferative DR may shift the balance between VEGF and connective tissue growth factor concentrations in the eye, which can result in fibrosis, retinal detachment, and 
vision loss. ${ }^{51,52}$ Therefore, knockdown of PLVAP expression, which attenuates caveolae formation and moderates hypoxia-induced vascular tortuosity, may provide a safer, endothelium-specific approach for DR and DME therapy. However, systemic inhibition of PLVAP potentially interferes with crucial functions of PLVAP in organ homeostasis. ${ }^{18,37,43}$ Because the eye has limited fluid exchange with other parts of the body, the preferred route for delivery of inhibitors of PLVAP would therefore be intravitreally by injection or implantation of slow-release drug delivery systems.

In conclusion, our study presents the first functional evidence that PLVAP plays a key role in BRB loss induced by VEGF and other factors and suggests that PLVAP regulates permeability in nonbarrier endothelia.

\section{Acknowledgments}

We thank Vincent Everts, Ph.D., (Academic Medical Center) for his assistance in electron microscopy, suggestions, and comments.

J.W.-K. designed and performed experiments, analyzed data, and wrote the manuscript; A.-E.v.d.W. performed experiments and edited the manuscript, I.M.C.V. performed experiments; I.K.; T.G.M.F.G., and D.V. contributed to the FA study; H.A.v.V. contributed to the EM study; I.K., C.J.F.V.N. and R.O.S. contributed to study design, discussion, and editing of the manuscript. I.K. is the guarantor of this work and, as such, had full access to all of the data in the study and takes responsibility for the integrity of the data and the accuracy of the data analysis.

\section{References}

1. Klaassen I, Van Noorden CJ, Schlingemann RO: Molecular basis of the inner blood-retinal barrier and its breakdown in diabetic macular edema and other pathological conditions. Prog Retin Eye Res 2013, 34: $19-48$

2. Witmer AN, Vrensen GF, Van Noorden CJ, Schlingemann RO: Vascular endothelial growth factors and angiogenesis in eye disease. Prog Retin Eye Res 2003, 22:1-29

3. Schlingemann RO, van Hinsbergh VW: Role of vascular permeability factor/vascular endothelial growth factor in eye disease. Br J Ophthalmol 1997, 81:501-512

4. Qaum T, Xu Q, Joussen AM, Clemens MW, Qin W, Miyamoto K, Hassessian H, Wiegand SJ, Rudge J, Yancopoulos GD, Adamis AP: VEGF-initiated blood-retinal barrier breakdown in early diabetes. Invest Ophthalmol Vis Sci 2001, 42:2408-2413

5. Abbott NJ, Ronnback L, Hansson E: Astrocyte-endothelial interactions at the blood-brain barrier. Nat Rev Neurosci 2006, 7: $41-53$

6. Wisniewska-Kruk J, Hoeben KA, Vogels IM, Gaillard PJ, Van Noorden CJ, Schlingemann RO, Klaassen I: A novel co-culture model of the blood-retinal barrier based on primary retinal endothelial cells, pericytes and astrocytes. Exp Eye Res 2012, 96: $181-190$

7. Armulik A, Genove G, Mae M, Nisancioglu MH, Wallgard E, Niaudet C, He L, Norlin J, Lindblom P, Strittmatter K, Johansson BR, Betsholtz C: Pericytes regulate the blood-brain barrier. Nature 2010, 468:557-561
8. Gesuete R, Orsini F, Zanier ER, Albani D, Deli MA, Bazzoni G, De Simoni MG: Glial cells drive preconditioning-induced blood-brain barrier protection. Stroke 2011, 42:1445-1453

9. Romero-Aroca P: Targeting the pathophysiology of diabetic macular edema. Diabetes Care 2010, 33:2484-2485

10. Wisniewska-Kruk J, Klaassen I, Vogels IM, Magno AL, Lai CM, Van Noorden CJ, Schlingemann RO, Rakoczy EP: Molecular analysis of blood-retinal barrier loss in the Akimba mouse, a model of advanced diabetic retinopathy. Exp Eye Res 2014, 122:123-131

11. Rakoczy EP, Ali Rahman IS, Binz N, Li CR, Vagaja NN, de Pinho M, Lai CM: Characterization of a mouse model of hyperglycemia and retinal neovascularization. Am J Pathol 2010, 177:2659-2670

12. Hammes HP, Feng Y, Pfister F, Brownlee M: Diabetic retinopathy: targeting vasoregression. Diabetes 2011, 60:9-16

13. Klaassen I, Hughes JM, Vogels IM, Schalkwijk CG, Van Noorden CJ, Schlingemann RO: Altered expression of genes related to blood-retina barrier disruption in streptozotocin-induced diabetes. Exp Eye Res 2009, 89:4-15

14. Argaw AT, Gurfein BT, Zhang Y, Zameer A, John GR: VEGFmediated disruption of endothelial CLN-5 promotes blood-brain barrier breakdown. Proc Natl Acad Sci U S A 2009, 106:1977-1982

15. Murakami T, Frey T, Lin C, Antonetti DA: Protein kinase cbeta phosphorylates occludin regulating tight junction trafficking in vascular endothelial growth factor-induced permeability in vivo. Diabetes 2012, 61:1573-1583

16. Antonetti DA, Barber AJ, Hollinger LA, Wolpert EB, Gardner TW: Vascular endothelial growth factor induces rapid phosphorylation of tight junction proteins occludin and zonula occluden 1. A potential mechanism for vascular permeability in diabetic retinopathy and tumors. J Biol Chem 1999, 274:23463-23467

17. Wang W, Dentler WL, Borchardt RT: VEGF increases BMEC monolayer permeability by affecting occludin expression and tight junction assembly. Am J Physiol Heart Circ Physiol 2001, 280: $\mathrm{H} 434-\mathrm{H} 440$

18. Schlingemann RO, Dingjan GM, Emeis JJ, Blok J, Warnaar SO, Ruiter DJ: Monoclonal antibody PAL-E specific for endothelium. Lab Invest $1985,52: 71-76$

19. Niemela H, Elima K, Henttinen T, Irjala H, Salmi M, Jalkanen S: Molecular identification of PAL-E, a widely used endothelial-cell marker. Blood 2005, 106:3405-3409

20. Stan RV, Tkachenko E, Niesman IR: PV1 is a key structural component for the formation of the stomatal and fenestral diaphragms. Mol Biol Cell 2004, 15:3615-3630

21. Stan RV: Structure of caveolae. Biochim Biophys Acta 2005, 1746 : 334-348

22. Schlingemann RO, Hofman P, Anderson L, Troost D, van der Gaag R: Vascular expression of endothelial antigen PAL-E indicates absence of blood-ocular barriers in the normal eye. Ophthalmic Res 1997, 29:130-138

23. Schlingemann RO, Hofman P, Vrensen GF, Blaauwgeers HG: Increased expression of endothelial antigen PAL-E in human diabetic retinopathy correlates with microvascular leakage. Diabetologia 1999 , 42:596-602

24. Carson-Walter EB, Hampton J, Shue E, Geynisman DM, Pillai PK, Sathanoori R, Madden SL, Hamilton RL, Walter KA: Plasmalemmal vesicle associated protein-1 is a novel marker implicated in brain tumor angiogenesis. Clin Cancer Res 2005, 11:7643-7650

25. Shue EH, Carson-Walter EB, Liu Y, Winans BN, Ali ZS, Chen J, Walter KA: Plasmalemmal vesicle associated protein-1 (PV-1) is a marker of blood-brain barrier disruption in rodent models. BMC Neurosci 2008, 9:29

26. Strickland LA, Jubb AM, Hongo JA, Zhong F, Burwick J, Fu L, Frantz GD, Koeppen H: Plasmalemmal vesicle-associated protein (PLVAP) is expressed by tumour endothelium and is upregulated by vascular endothelial growth factor-A (VEGF). J Pathol 2005, 206: 466-475 
27. Schlingemann RO, Bots GTAM, Van Duinen SG, Ruiter DJ: Differential expression of endothelium-specific antigen PAL-E in vasculature of brain tumors and preexistent brain capillaries. Ann N Y Acad Sci 1988, 529:111-114

28. Hofman P, Blaauwgeers HG, Vrensen GF, Schlingemann RO: Role of VEGF-A in endothelial phenotypic shift in human diabetic retinopathy and VEGF-A-induced retinopathy in monkeys. Ophthalmic Res 2001, 33:156-162

29. Junge HJ, Yang S, Burton JB, Paes K, Shu X, French DM, Costa M, Rice DS, Ye W: TSPAN12 regulates retinal vascular development by promoting Norrin- but not Wnt-induced FZD4/beta-catenin signaling. Cell 2009, 139:299-311

30. Wang Y, Rattner A, Zhou Y, Williams J, Smallwood PM, Nathans J: Norrin/Frizzled4 signaling in retinal vascular development and blood brain barrier plasticity. Cell 2012, 151:1332-1344

31. Liebner S, Corada M, Bangsow T, Babbage J, Taddei A, Czupalla CJ, Reis M, Felici A, Wolburg H, Fruttiger M, Taketo MM, von Melchner H, Plate KH, Gerhardt H, Dejana E: Wnt/beta-catenin signaling controls development of the blood-brain barrier. J Cell Biol 2008, 183:409-417

32. Daneman R, Zhou L, Kebede AA, Barres BA: Pericytes are required for blood-brain barrier integrity during embryogenesis. Nature 2010, 468:562-566

33. Witmer AN, van Blijswijk BC, van Noorden CJ, Vrensen GF, Schlingemann RO: In vivo angiogenic phenotype of endothelial cells and pericytes induced by vascular endothelial growth factor-A. J Histochem Cytochem 2004, 52:39-52

34. Andersen CL, Jensen JL, Orntoft TF: Normalization of real-time quantitative reverse transcription-PCR data: a model-based variance estimation approach to identify genes suited for normalization, applied to bladder and colon cancer data sets. Cancer Res 2004, 64:5245-5250

35. Smith LE, Wesolowski E, McLellan A, Kostyk SK, D'Amato R, Sullivan R, D'Amore PA: Oxygen-induced retinopathy in the mouse. Invest Ophthalmol Vis Sci 1994, 35:101-111

36. McDowell EM, Trump BF: Histologic fixatives suitable for diagnostic light and electron microscopy. Arch Pathol Lab Med 1976, 100:405-414

37. Stan RV, Arden KC, Palade GE: cDNA and protein sequence, genomic organization, and analysis of cis regulatory elements of mouse and human PLVAP genes. Genomics 2001, 72:304-313

38. Witmer AN, Blaauwgeers HG, Weich HA, Alitalo K, Vrensen GF, Schlingemann RO: Altered expression patterns of VEGF receptors in human diabetic retina and in experimental VEGF-induced retinopathy in monkey. Invest Ophthalmol Vis Sci 2002, 43:849-857

39. Murakami T, Felinski EA, Antonetti DA: Occludin phosphorylation and ubiquitination regulate tight junction trafficking and vascular endothelial growth factor-induced permeability. J Biol Chem 2009 , 284:21036-21046

40. Feng Y, Venema VJ, Venema RC, Tsai N, Behzadian MA, Caldwell RB: VEGF-induced permeability increase is mediated by caveolae. Invest Ophthalmol Vis Sci 1999, 40:157-167
41. Lin ZX, Yang LJ, Huang Q, Lin JH, Ren J, Chen ZB, Zhou LY, Zhang PF, Fu J: Inhibition of tumor-induced edema by antisense VEGF is mediated by suppressive vesiculo-vacuolar organelles (VVO) formation. Cancer Sci 2008, 99:2540-2546

42. Hofman P, Blaauwgeers HG, Tolentino MJ, Adamis AP, Nunes Cardozo BJ, Vrensen GF, Schlingemann RO: VEGF-A induced hyperpermeability of blood-retinal barrier endothelium in vivo is predominantly associated with pinocytotic vesicular transport and not with formation of fenestrations. Vascular endothelial growth factor-A. Curr Eye Res 2000, 21:637-645

43. Stan RV, Tse D, Deharvengt SJ, Smits NC, Xu Y, Luciano MR, McGarry CL, Buitendijk M, Nemani KV, Elgueta R, Kobayashi T, Shipman SL, Moodie KL, Daghlian CP, Ernst PA, Lee HK, Suriawinata AA, Schned AR, Longnecker DS, Fiering SN, Noelle RJ, Gimi B, Shworak NW, Carrière C: The diaphragms of fenestrated endothelia: gatekeepers of vascular permeability and blood composition. Dev Cell 2012, 23:1203-1218

44. Olsson AK, Dimberg A, Kreuger J, Claesson-Welsh L: VEGF receptor signalling - in control of vascular function. Nat Rev Mol Cell Biol 2006, 7:359-371

45. Tahir SA, Park S, Thompson TC: Caveolin-1 regulates VEGFstimulated angiogenic activities in prostate cancer and endothelial cells. Cancer Biol Ther 2009, 8:2286-2296

46. Santibanez JF, Blanco FJ, Garrido-Martin EM, Sanz-Rodriguez F, del Pozo MA, Bernabeu C: Caveolin-1 interacts and cooperates with the transforming growth factor-beta type I receptor ALK1 in endothelial caveolae. Cardiovasc Res 2008, 77:791-799

47. Dejana E, Orsenigo F, Lampugnani MG: The role of adherens junctions and VE-cadherin in the control of vascular permeability. J Cell Sci 2008, 121:2115-2122

48. Nagy JA, Dvorak AM, Dvorak HF: Vascular hyperpermeability, angiogenesis, and stroma generation. Cold Spring Harb Perspect Med 2012, 2:a006544

49. Carmeliet P, Storkebaum E: Vascular and neuronal effects of VEGF in the nervous system: implications for neurological disorders. Semin Cell Dev Biol 2002, 13:39-53

50. Saint-Geniez M, Maharaj AS, Walshe TE, Tucker BA, Sekiyama E, Kurihara T, Darland DC, Young MJ, D’Amore PA: Endogenous VEGF is required for visual function: evidence for a survival role on muller cells and photoreceptors. PLoS One 2008, 3:e3554

51. Kuiper EJ, Van Nieuwenhoven FA, de Smet MD, van Meurs JC, Tanck MW, Oliver N, Klaassen I, Van Noorden CJ, Goldschmeding R, Schlingemann RO: The angio-fibrotic switch of VEGF and CTGF in proliferative diabetic retinopathy. PLoS One 2008, 3:e2675

52. Van Geest RJ, Lesnik-Oberstein SY, Tan HS, Mura M, Goldschmeding R, Van Noorden CJ, Klaassen I, Schlingemann RO: A shift in the balance of vascular endothelial growth factor and connective tissue growth factor by bevacizumab causes the angiofibrotic switch in proliferative diabetic retinopathy. Br J Ophthalmol 2012, 96:587-590 